\title{
Dynamics of Low-Density Ultracold Rydberg Gases
}

\author{
J. O. Day, E. Brekke, and T. G. Walker* \\ Department of Physics, University of Wisconsin-Madison, Madison, WI 53706
}

(Dated: October 31, 2018)

\begin{abstract}
Population dynamics in weakly-excited clouds of ultracold ${ }^{87} \mathrm{Rb}$ Rydberg atoms were studied by means of trap loss, fluorescence detection, and state dependent stimulated emission. Rydberg atoms were excited to various $n l$ Rydberg states via continuous two-photon excitation from a magnetooptical trap. A stimulated emission probe laser was then used to bring the Rydberg atoms down to the $6 \mathrm{P}_{3 / 2}$ state, allowing state-dependent detection of the Rydberg atoms. Measurements of trap loss and fluorescent emission reveal information about the evolution of the Rydberg populations. In particular, population in the initial Rydberg state quickly transfers to other Rydberg states by a non-collisional mechanism, likely superradiant emission. The trap-loss measurements are consistent with black-body ionization as the dominant loss mechanism.
\end{abstract}

PACS numbers: $32.80 . \mathrm{Ee}, 42.50 . \mathrm{Nn}, 34.50 . \mathrm{Cx}$

In the past 10 years it has become possible to study the interesting properties of dense clouds of ultracold Rydberg atoms, usually produced by laser excitation from magneto-optical traps (MOTs) [1, 2]. A variety of interesting collisional phenomena [3, 4, 5, 6, 7, 8, 9, 10, 11. have been observed, including surprisingly rapid spontaneous conversion of dense ultracold Rydberg clouds into plasmas [8, 12, 13]. It is clear that resonant [2, 4, , 5, 6, 6] and near resonant energy transfer collisions [8, 9, 10] play important roles in the population dynamics of clouds of ultracold Rydberg atoms.

In addition, intriguing proposals to use the strong, long-range interactions between ultracold Rydberg atoms to perform conditional quantum manipulations [14] are driving a number of groups to study coherent manipulations with small numbers of atoms and small samples. In addition, the concept of Rydberg blockade [15] brings up the possibility of using spatially confined samples of atoms [16, 17] for mesoscopic quantum manipulations. Using such collective Rydberg excitations might allow novel quantum applications such as fast quantum gates 15, 18], single atom and directed single photon sources [19], and fast quantum state detection [20].

The ultracold Rydberg cloud experiments, with many Rydberg atoms excited at once, and the quantum manipulation experiments, with only one Rydberg atom excited at a time, operate in very different regimes. Still, it is interesting to note that in both cases their success is reliant on the strong interatomic forces between Rydberg atoms. For quantum manipulations, one wants the Rydberg-Rydberg interactions to be so strong that multi-atom excitations are suppressed [21]. A number of experiments [22, 23, 24, 25] report suppression signatures suggesting that this regime should be achievable. Most recently, collisional dephasing of coherent Rydberg Rabi flopping was observed for only two atoms confined in a dipole trap [26], and dephasing of coherent Rydberg

*tgwalker@wisc.edu excitation in a BEC was also observed 27].

The experiment reported in this paper studies a transition regime between the two types of experiments. We weakly excite ultracold Rydberg atoms in an unblockaded regime and study their evolution using three tools: trap loss, spontaneous emission, and stimulated emission. Using these diagnostics, we find that under our weak excitation conditions the probability is very small that the Rydberg atoms experience an inelastic collision sufficiently strong to leave the trap. They predominantly return to the atomic ground state by spontaneous emission. Using the stimulated emission diagnostic, we also observe rapid population transfer out of the initial Rydberg state-the initial Rydberg state is depopulated on a time scale substantially shorter than expected for transfer from black-body radiation or single-atom spontaneous emission. The transfer rate slowly decreases with increasing principal quantum number, strongly suggesting that the transfer process is not collisional in nature. We build upon the recent observation of superradiance by Wang et al. 28] and argue that superradiance is the likely mechanism for this transfer process. Finally, we show that trap loss rates are consistent with expectations from blackbody ionization. We conclude with implications of these results for other experiments.

\section{TRAP LOSS STUDIES}

In this section, we describe observations of loss of atoms from the MOT due to Rydberg excitation. Other groups [23, 29] have reported observations of Rydberg trap loss under various conditions, without attempting to explain the mechanisms for trap loss, especially under the low excitation conditions reported here. We find that trap loss rates are much less than excitation rates, showing that, once excited, the Rydberg atoms primarily decay back to the ground state by spontaneous emission processes.

We excite magneto-optically trapped ${ }^{87} \mathrm{Rb} n l_{j}$ Rydberg atoms using continuous-wave $5 \mathrm{~S}_{1 / 2} \rightarrow 5 \mathrm{P}_{3 / 2} \rightarrow \mathrm{nl}_{j}$ 


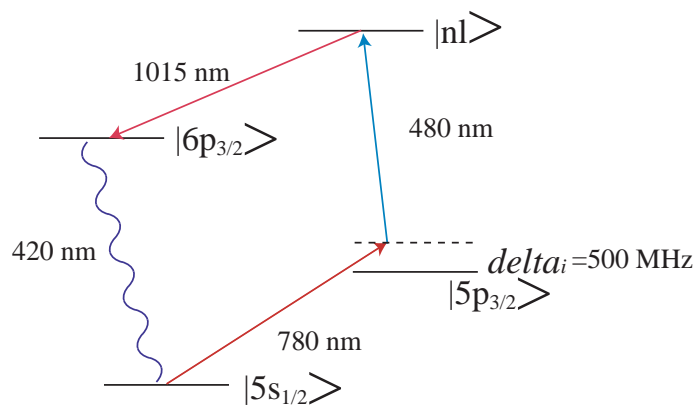

FIG. 1: Diagram of the Rydberg excitation scheme.

two-photon excitation. The $780 \mathrm{~nm}(20 \mathrm{~mW})$ and $480 \mathrm{~nm}$ $(10 \mathrm{~mW})$ lasers used are tuned $\delta_{i}=500 \mathrm{MHz}$ above the intermediate $5 \mathrm{P}_{3 / 2} \mathrm{~F}=3$ state, thus avoiding direct excitation of the $5 \mathrm{P}_{3 / 2}$ state (Fig. 1). Both excitation beams have spatial dimensions on the order of the $\sim 1 \mathrm{~mm}$ size of the MOT. Typical two-photon excitation rates vary from 10 to 100/s depending on $\mathrm{n}$ level. These rates are deduced from calculated atomic matrix elements, measured laser intensities, and the observed transition line-widths, and are averaged over the atomic and laser spatial distributions. The effective excitation rate is $\left|\epsilon_{2}\right|^{2} / \Delta$, where $\epsilon_{2}=\epsilon_{r} \epsilon_{b} / 4 \delta_{i}$ is the two-photon Rabi frequency, $\epsilon_{r}$ and $\epsilon_{b}$ are the single photon Rabi frequencies for the $780 \mathrm{~nm}$ and $480 \mathrm{~nm}$ lasers, and $\Delta$ is the observed transition linewidth which is typically 8 to $10 \mathrm{MHz}$ for these scans as described below. This results in $\sim 10^{4}$ Rydberg atoms at a relatively low density of $10^{7} / \mathrm{cm}^{3}$. This density is an order of magnitude or more smaller than the densities at which ultracold plasmas are formed [8, 13]. By changing the frequency of the $480 \mathrm{~nm}$ laser, we excite to Rydberg states at $28 \mathrm{D}, 43 \mathrm{D}, 58 \mathrm{D}$, and $30 \mathrm{~S}$; in most cases the Dstate excitations are to the $\mathrm{J}=5 / 2$ state. These particular states are chosen to provide a range of different spontaneous decay rates (which scale roughly as $\sim n^{-3}$ ) and a wider range of Rydberg-Rydberg van der Waals interactions $C_{6} R^{-6}$, with $C_{6}(28,43,58) \approx(0.08,540,330) \mathrm{GHz}$ $\mu \mathrm{m}^{6}[21]$.

A very simple and effective way to observe excitation of Rydberg atoms in a MOT is via trap loss, with a resulting decrease in the observed fluorescence from the MOT. Processes such as black-body ionization, photoionization by the applied lasers, and inelastic collisions between Rydberg atoms and ground state atoms either ionize the atoms or give the atoms sufficient (>10 K) kinetic energy to leave the trap even if they radiatively return to the ground state. Radiative processes such as photon emission or absorption, elastic or inelastic collisions with cold electrons, and near-resonant RydbergRydberg collisions do not transfer enough kinetic energy to the atoms to cause trap loss. Trap loss is very sensitive; it is easy to observe a $0.1 / \mathrm{s}$ change in the trap loss rate from the MOT. With modest Rydberg excitation rates of $10 / \mathrm{s}$, a $1 \%$ inelastic or photo-ionization channel

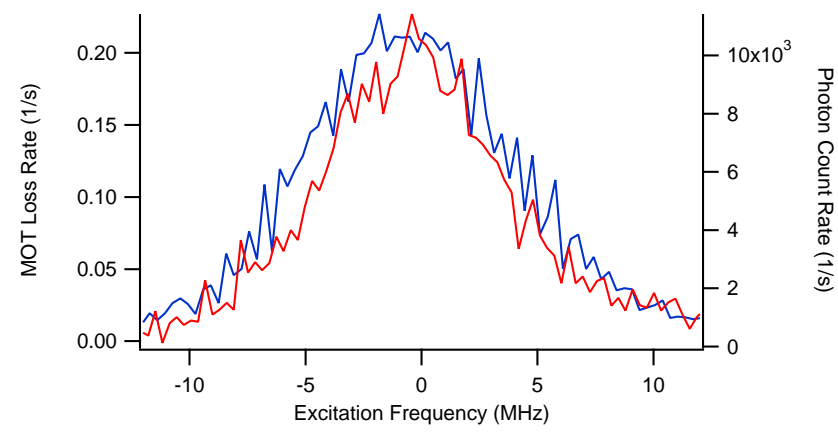

FIG. 2: Excitation beams are scanned over the Rydberg resonance at the $28 \mathrm{D}$ state. The peak excitation rate is $110 / \mathrm{s}$. The figure shows the loss rate from the trap induced by the excitation beams as well as the photon count rate from the spontaneous emission cascade.

can easily be detected.

Since the excitation lasers are sufficiently off-resonance to cause negligible perturbations to the MOT trapping and cooling processes, the loss due to production of Rydberg atoms affects the MOT only through a change in the ejection or loss rate from the trap. If the MOT lasers produce a loading rate $L$ and a loss rate $\Gamma_{0}$, the steady-state number of trapped atoms is $N_{g 0}=L / \Gamma_{0}$. Excitation of Rydberg atoms using lasers tuned to frequency $\nu$ adds a new loss rate $\Gamma_{1}$ which then changes the number of atoms to $N_{g}(\nu)$. The loss rate can then be found from

$$
\Gamma_{1}(\nu)=\Gamma_{0}\left(\frac{N_{g 0}}{N_{g}(\nu)}-1\right)
$$

This is operationally simpler than taking separate MOT loading transients at each frequency and determining the loss rate from the time constant. We have checked that the two procedures give the same results.

Figure 2 shows the results of sweeping the two-photon excitation frequency through a typical Rydberg resonance. Loss rates induced by the excitation lasers are around $0.2 / \mathrm{s}$, a rate much smaller than the $110 / \mathrm{s}$ Rydberg excitation rate. We may infer from this that the probability of inelastic collisions occurring with sufficient energy transfer for the atoms to leave the trap is less than $1 / 500$. For the other states studied, we find $1 / 50$ for $43 \mathrm{D}$ and $58 \mathrm{D}$, and $1 / 1000$ for the $30 \mathrm{~S}$ state.

Noting that the trap loss probabilities are significantly higher for $43 \mathrm{D}$ and $58 \mathrm{D}$ as compared to $28 \mathrm{D}$, a logical hypothesis would be that inelastic collisions are responsible for the trap loss. To this end, we compared the trap loss rates from $41 \mathrm{D}$ and $43 \mathrm{D}$, and found little difference. Since the 43D van der Waals interactions are anomalously large due to a near resonance in the $41 \mathrm{P}+45 \mathrm{~F}$ and $43 \mathrm{D}+43 \mathrm{D}$ potentials [7, 21, 30], then very different results should be obtained when comparing 41D and 43D if collisions were responsible. Thus we again find no evidence that resonant energy transfer collisions cause significant trap loss. 
The following estimate supports these conclusions. The inelastic collision rate should be a capture rate multiplied by a probability of energy transfer. We estimate the capture rate as $\eta v \sigma$, where $\sigma=\pi R_{0}^{2}, C_{6} R^{-6}=k T$, and $\eta v$ is the Rydberg atom flux. We then deduce a capture rate of

$$
\eta v \sigma=\eta v \pi\left(C_{6} / k T\right)^{1 / 3} \sim 200 / s
$$

for an $n=43$ density of $\eta=10^{7} / \mathrm{cm}^{3}$. This rate, already small, is further reduced by the energy transfer probability. For states like 43D, where the van der Waals interactions are repulsive at long range, there are no thermally accessible curve crossings and we expect the rate to be suppressed by at least a Boltzmann factor $e^{-\Delta E / k T}$, which even for $43 \mathrm{D}$ with small $\Delta E$ is a factor of $\sim 10^{-2}$.

The line-widths observed are significantly broader than the laser line-widths, and exceed what we expect from drifts of our reference cavity used to stabilize the $480 \mathrm{~nm}$ laser frequency. This is consistent with observations by others [23, 31].

Since the atoms are only rarely leaving the trap due to Rydberg-Rydberg collision or ionization, they must primarily return to the ground state by emission of one or more photons. In the next section we introduce a direct probe of the Rydberg state population that will give more information about the details of this process.

\section{CASCADE FLUORESCENCE}

As a complementary diagnostic to trap loss observations, we use a photon counting module and a narrowband interference filter to detect the $420 \mathrm{~nm}$ decay photons from the $6 \mathrm{P}_{3 / 2}$ state to the $5 \mathrm{~S}_{1 / 2}$ ground state. These photons are only observed coming from the MOT cloud under conditions of Rydberg excitation. The fluorescent branching ratio to the $6 \mathrm{P}_{3 / 2}$ from a high $n \mathrm{~S}$ or $n \mathrm{D}$ Rydberg state is calculated to be about $b_{r}=0.15$, varying only slightly with principal quantum number. (The predominant channel is emission to the $5 \mathrm{P}_{3 / 2}$ state due to its much larger $\omega^{3}$ factor in the emission rate, which easily compensates for a slightly smaller dipole matrix element. This channel is difficult to observe in the presence of strong MOT fluorescence at the same wavelength.) The probability of multiple photon cascade into the $6 \mathrm{P}_{3 / 2}$ state is small because the long-wavelength photons required are disfavored. $\mathrm{P}$ and $\mathrm{F}$ Rydberg states predominantly cascade into $5 \mathrm{~S}, 6 \mathrm{~S}$, and $4 \mathrm{D}$ levels, all of which lie energetically below the $6 \mathrm{P}$ state. Even for states slightly above the $6 \mathrm{P}$, such as the $5 \mathrm{D}$, the predominant decay channel is to states below the $6 \mathrm{P}$. Thus the cascade fluorescence is likely a reliable probe of the $\mathrm{S}$ or $\mathrm{D}$ Rydberg state populations, and is relatively insensitive to P or F Rydberg states. Figure 2 shows the observed cascade counts as the excitation lasers are scanned across a Rydberg resonance.

The observed cascade signal has a background, mainly from dark counts, of around $250 / \mathrm{s}$ and reaches a peak signal of about $10000 / \mathrm{s}$ for the $28 \mathrm{D}$ excitation. We can compare these observed rates with our expected rates from the calculated excitation rates and fluorescent branching ratios. Accounting for the finite collection solid angle $\Omega=3 \times 10^{-3}, \eta=3.4 \%$ detection efficiency of the photomultiplier tube, and a calculated $6 \mathrm{P}_{3 / 2}-5 \mathrm{~S}_{1 / 2}$ emission branching ratio $b_{6}=0.31$ we expect to observe a cascade count rate

$$
c_{6}=R_{2} N_{g} b_{r} b_{6} \eta \Omega \frac{A_{r}}{A_{r}+A_{B B}}=18,000 / \mathrm{s}
$$

at the peak of the $28 \mathrm{D}$ excitation resonance. The ratio of spontaneous to total decay rates accounts for the effects of black-body radiation causing radiative transitions to nearby Rydberg states. These states are assumed, by the argument above, not to result in detected cascade photons. For the data seen in Fig. 2 we observe 10000/s, only $55 \%$ of the expected count rate. This suggests that roughly $1 / 2$ of the Rydberg atoms are being transferred out of the excitation state by some other process. The ratio of expected to detected cascade counts for the other excitation states $(30 \mathrm{~S}, 43 \mathrm{D}$, and $58 \mathrm{D})$ are $(0.5,0.6,0.6)$. Again, by the arguments from the previous section, the explanation for this cannot be inelastic Rydberg-Rydberg collisions, which would either produce an extremely large trap loss rate in contradiction to observations, or would necessarily vary greatly with principal quantum number.

\section{STIMULATED EMISSION PROBE}

For dipole blockade applications, one is particularly interested in the evolution of the blockaded Rydberg state. Furthermore, in the particular applications of dipole blockade to single atom and single photon sources [19], stimulated emission is used to couple the blockaded Rydberg level to an intermediate atomic level. Thus it is natural for us to pursue the development of a stimulated emission probe of Rydberg dynamics. The intensity dependence of the signals produced by the stimulated emission probe allows further information to be obtained about the population dynamics. Such a probe is nondestructive, and has inherently high spectral resolution. While being less general and less sensitive than field ionization, it does have the potential to be applied to a number of different states, subject to dipole selection rules.

As shown in Fig. 1 we apply a tunable diode laser in the range of 1013 to $1027 \mathrm{~nm}$ to perform stimulated emission probing of the Rydberg states produced by the twophoton excitation. This "state probe" de-excites atoms from the Rydberg states to the $6 \mathrm{P}_{3 / 2}$ state, which subsequently decays via a $420 \mathrm{~nm}$ photon to the $5 \mathrm{~S}$ ground state. The $6 \mathrm{P}_{3 / 2}$ state is either detected in the same manner as the cascade light mentioned above, or by a reduction in trap loss (explained below). As with the excitation lasers, the spatial size of the state-probe laser is comparable to the MOT cloud size. We deliver nearly 100 $\mathrm{mW}$ of light to the atoms, corresponding to stimulated 


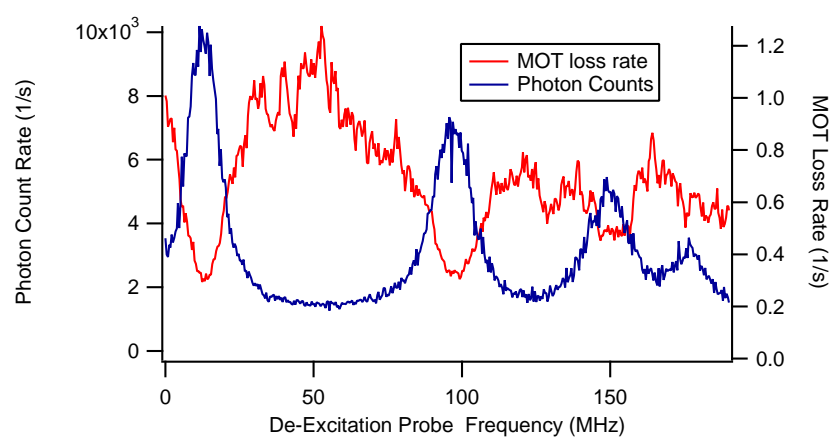

FIG. 3: Stimulated emission probe scan across the $6 \mathrm{P}_{3 / 2}$ manifold. The cascade count background is around 1000 counts.

emission rates from $5 \times 10^{5}$ to $5 \times 10^{6} / \mathrm{s}$ depending upon the Rydberg level involved. The natural decay rate of the $6 \mathrm{P}_{3 / 2}$ level of $9.2 \times 10^{6} / \mathrm{s}$ is fast enough that population cannot accumulate there. The state-probe laser is stabilized to a $300 \mathrm{MHz}$ optical spectrum analyzer that is itself locked to a $780 \mathrm{~nm} \mathrm{Rb}$ saturated absorption resonance. It is tuned by changing the frequency of a double-passed acousto-optic modulator.

When the stimulated emission probe is on resonance, atoms are returned to the ground state more quickly than they would otherwise spontaneously radiate from the long-lived Rydberg levels. This reduces the loss rate from the trap as the Rydberg atoms do not stay excited long enough for loss mechanisms such as black-body ionization and inelastic collisions to remove many of them from the trap. Keeping the excitation beams on resonance with the two-photon excitation, we can scan the frequency of the stimulated emission probe to observe the $6 \mathrm{P}_{3 / 2}$ hyperfine manifold. Such a scan is shown in Fig. 3. When tuned on resonance with the $6 \mathrm{P}_{3 / 2} \mathrm{~F}=3$ hyperfine state, the MOT loss rate is reduced from $0.8 / \mathrm{s}$ to $0.2 / \mathrm{s}$, with the amount of reduction depending on probe intensity.

The state-probe laser produces a reduction in the trap loss rates. An example of this for the $28 \mathrm{D}_{5 / 2}$ Rydberg state is shown in Fig. 4 There the trap loss is shown as a function of the stimulated emission rate from the probe beam. The figure clearly demonstrates that by using a sufficiently high state-probe intensity the atoms can be returned to the ground state before ionization or inelastic collisions can occur, thus reducing the loss rate of the MOT. The surprising feature of the data is that much higher intensities are needed than would be expected if the dominant population transfer from the Rydberg level were spontaneous decay or black-body transfer (rates of $4 \times 10^{4} / \mathrm{s}$ and $2 \times 10^{4} / \mathrm{s}$, respectively) [32]. The residence time of the Rydberg atoms is deduced to be nearly 10 $\mu \mathrm{s}$.

Alternatively, we can measure the state probe laser effects by detecting the number of $6 \mathrm{P}_{3 / 2}$ decay photons from stimulated emission from the Rydberg state. The count rate data, scaled by the number of ground state

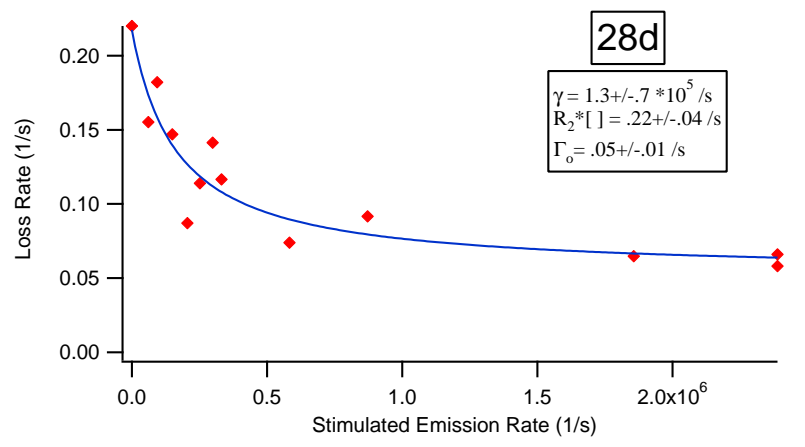

FIG. 4: Loss rate dependence on stimulated emission probe intensity for the $28 \mathrm{D}$ state, showing short residence times for the Rydberg state produced by two-photon excitation.

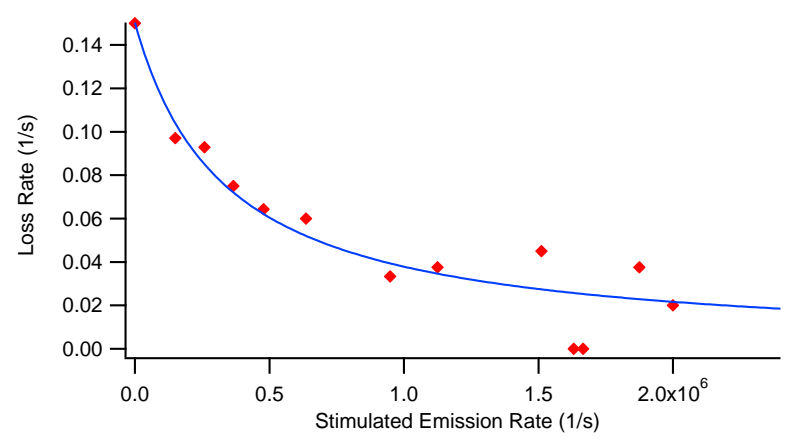

FIG. 5: Loss rate dependence on stimulated emission probe intensity for the $28 \mathrm{D}$ state, with the MOT magnetic field switched off during excitation.

MOT atoms, is shown in Fig. 6 for the 28D state. The cascade and background signal have been subtracted for this data, thus there are no counts when the stimulated emission rate is zero. As with the repletion data of Fig. 4, the counts saturate at a stimulated emission rate of around $1 \times 10^{5} / \mathrm{s}$, which implies that the other rates out of the excitation state must be on this order.

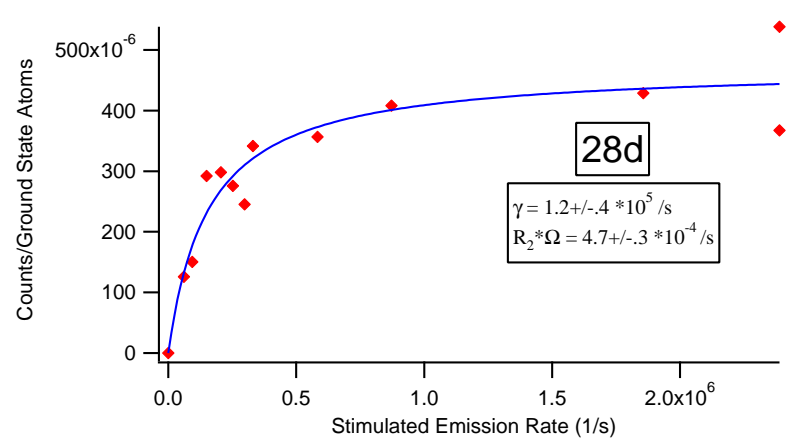

FIG. 6: Dependence of $6 \mathrm{P}_{3 / 2}$ decay counts on stimulated emission probe intensity for the $28 \mathrm{D}$ state.

From the trap loss or state probe data, the residence time of the Rydberg state varies from about $10 \mu$ s at $n=$ 28 to about $50 \mu$ s at $n=58$. Thus the population is being 
transferred out of the initial Rydberg state faster than can be accounted for by spontaneous decay and blackbody transfer (decay times of $25 \mu \mathrm{s}$ and $50 \mu \mathrm{s}$ at $n=28$, respectively). The transfer times increase with $n$, just the opposite dependence as would be expected for energy transfer collisions between Rydberg states. These would be expected to decrease with $n$ due to rapid increase in van der Waals interaction strengths. This counter trend thus implies that Rydberg-Rydberg collisions are not the process responsible for the fast transfer out of the initial Rydberg state.

Interestingly, the 30S state has the highest transfer rate to other Rydberg states. This again implies that atom-atom interactions do not dominate the process, as the strengths of van der Waals interactions tend to be larger for D states than for $\mathrm{S}$ states.

We have checked that the transfer rates increase with increasing excitation rate. This confirms that some transfer process besides black-body radiation is occurring.

As can be seen from Fig. 4, there is a non-zero loss rate at high probe intensities where the stimulated emission laser should fully deplete the original excitation state. There are several possible explanations for this. If there were a loss mechanism that was being enhanced by the state-probe laser, this would produce such a behavior. However, processes such as photoionization or light-induced $6 \mathrm{P}-5 \mathrm{~S}$ collisions can be estimated to be far too weak to account for this effect. Another possibility has to do with Zeeman precession in the nd states causing population to accumulate in inaccessible magnetic sublevels. The magnetic field gradient used to confine the MOT atoms is large enough that precession between magnetic sublevels of the Rydberg atoms at the edges of the MOT occurs at a rate of several $\mathrm{MHz}$, which is on the order of the stimulated emission rate. The result of this precession is that a fraction of the atoms - up to $1 / 3$ - move to a state that is inaccessible to the state-probe laser because of the dipole selection rules determined by the polarization of the state-probe laser. For linearly polarized state-probe light, $m= \pm 5 / 2$ Zeeman levels cannot be excited to the $6 \mathrm{P}_{3 / 2}$ state. Thus population that accumulates in these levels cannot be de-excited by the state-probe laser.

This effect was verified by repeating the experiment with the MOT magnetic field being switched off for 10 ms intervals and only switching the Rydberg excitation lasers during the times the field was off. This data, shown in Fig. 5 for $n=28$ shows that Rydberg populations at high state probe intensities are markedly reduced as compared to when the magnetic field is on. The reduced signal-to-noise for this experiment made it possible to do this only for $n=28$.

\section{MODEL OF RYDBERG POPULATION DYNAMICS}

In the previous sections, we have described the basic processes that are evidently at work under the conditions of our experiment, and their experimental signatures. To further analyze the results, we present here a simplified model of the Rydberg dynamics and use it to extract the values of a few simple parameters from the data. Confirming the interpretation in the previous section, we find that some process that does not cause trap loss nevertheless transfers population out of the excitation Rydberg state on a time scale short compared to spontaneous or black-body lifetimes. The lengthening of this time scale with principal quantum number leads us to believe that it is not inelastic collisions between Rydberg atoms.

The processes included in the model are illustrated in Fig. 7 We describe the system with a three state model: A ground state $|g\rangle$ with $N_{g}$ atoms, the excitation Rydberg state $|r\rangle$ with $N_{r}$ atoms, and an additional effective Rydberg state $|s\rangle$ that accounts for other states that are populated from state $|r\rangle$.

The dynamics of the excitation state $|r\rangle$ depend on laser excitation and de-excitation, spontaneous and black-body radiation, and transfer to the other Rydberg states $|s\rangle$. Population enters $|r\rangle$ by excitation from the ground state at a rate $R_{2} N_{g}$ where $R_{2}$ is calculated as described in Section [1. Spontaneous decay to low-lying levels occurs at a rate $A_{r} N_{r}$. Black-body radiation and other potential processes that transfer atoms to other Rydberg states occur at a rate $\gamma N_{r}$. There is also the possibility of trap loss (through ionization, for example) at a rate $\Gamma_{r}$ directly from state $|r\rangle$. Finally, de-excitation from the state-probe laser occurs at a rate $R_{3} N_{r}$. Thus

$$
\frac{d N_{r}}{d t}=R_{2} N_{g}-A_{r} N_{r}-R_{3} N_{r}-\gamma N_{r}-\Gamma_{r} N_{r}
$$

is the rate equation for the excitation state population.

The other Rydberg states are produced by collisional or radiative transfer from state $|r\rangle$ at the rate $\gamma N_{r}$ and have an effective radiative lifetime $A_{s}$. We also assume that these states can cause trap loss at a rate $\Gamma_{s}$ due to black-body ionization and other collisional processes. Thus they obey

$$
\frac{d N_{s}}{d t}=\gamma N_{r}-\Gamma_{s} N_{s}-A_{s} N_{s}
$$

We are assuming that transfer from $|s\rangle$ back to $|r\rangle$ is unlikely.

In addition to the radiative de-excitation and excitation processes with the Rydberg levels, the ground state population $N_{g}$ is affected by MOT loading $(L)$ and loss $\left(\Gamma_{0}\right)$ processes that we assume are not materially changed when the Rydberg excitation lasers are on. The resulting rate equation for the ground state population is

$$
\frac{d N_{g}}{d t}=L-\Gamma_{0} N_{g}-R_{2} N_{g}+\left(A_{r}+R_{3}\right) N_{r}+A_{s} N_{s}
$$




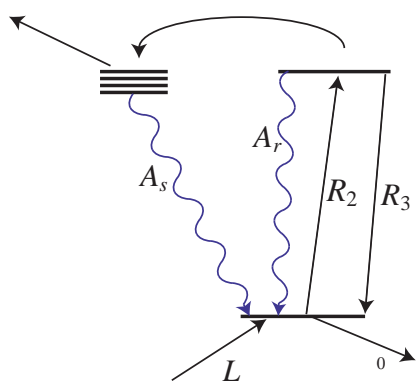

FIG. 7: Diagram of the simplified model of Rydberg population dynamics.

Plugging in the steady-state solutions to Eq. 4 and 5 gives

$$
\frac{d N_{g}}{d t}=L-\Gamma_{0} N_{g}-\Gamma N_{g}
$$

The loss-rate from the trap is increased by an amount

$$
\Gamma \approx \frac{R_{2} \gamma}{A_{r}+R_{3}+\gamma}\left[\frac{\Gamma_{s}}{A_{s}}+\frac{\Gamma_{r}}{\gamma}\right]
$$

This is a product of two factors. The first essentially measures the excitation rate of Rydberg atoms, modified by the de-excitation due to the state-probe laser. The second factor is the probability that the excited Rydberg atoms experience trap loss as opposed to radiatively decaying back to the ground state.

The model similarly predicts the count rate of $420 \mathrm{~nm}$ photons produced by the state-probe laser:

$$
\frac{I_{3}}{N_{g}}=\frac{R_{3} R_{2} \Omega \eta b_{r} b_{6}}{A_{r}+R_{3}+\gamma}
$$

Thus the state-probe-induced $420 \mathrm{~nm}$ count rate can also be used to determine the transfer rate $\gamma$, with the data and fit for 28D shown in Fig. 6.

The results of fitting our experimental data to this model are summarized in Table I]. We list there the deduced values of the primary unknowns $\gamma$ and $\Gamma_{s}$, as well as the assumed values for the input parameters $A_{r}$ and $A_{s}$. We note that both trap loss and $420 \mathrm{~nm}$ count rates can be used to extract $\gamma$, an important internal consistency check. The primary results are these:

- The atoms transfer out of the excitation Rydberg state at a rate $\gamma$ that is substantially faster than spontaneous decay or black-body transfer rates.

- The mechanism for population transfer, to an excellent approximation, does not cause trap loss.

- The population transfer rate decreases slowly with increasing principle quantum number, as opposed to the expected rapid increase if near-resonant energy transfer collisions were the relevant mechanism.
- The probability of trap loss is very small; most Rydberg excitations result in radiative repopulation of the ground state without trap loss.

These conclusions from fitting the experimental data to the model are consistent with the simplified analyses presented in Sections 【III In the next two sections we will discuss their implications.

TABLE I: A summary of transfer rate data using both count rate and loss rate methods. As a basis for comparison, the black-body transfer rate $\mathrm{A}_{B B}$, spontaneous emission rate $\mathrm{A}_{r}$, the estimated mean spontaneous emission rate $A_{s}$ from other Rydberg states, and the inferred trap loss rate $\Gamma_{s}$ are also included. All rates are in units of $\mathrm{s}^{-1}$.

\begin{tabular}{ccccccc}
\hline \hline State & $\gamma$ (counts) & $\gamma($ loss $)$ & $A_{B B}$ & $A_{r}$ & $A_{s}$ & $\Gamma_{s}$ \\
\hline $28 \mathrm{D}$ & $1.2 \times 10^{5}$ & $1.3 \times 10^{5}$ & $2.6 \times 10^{4}$ & $4.1 \times 10^{4}$ & $3.1 \times 10^{4}$ & 265 \\
$43 \mathrm{D}$ & $7.4 \times 10^{4}$ & $7.2 \times 10^{4}$ & $1.1 \times 10^{4}$ & $1.1 \times 10^{4}$ & $2.0 \times 10^{4}$ & 602 \\
$58 \mathrm{D}$ & $2.6 \times 10^{4}$ & $2.0 \times 10^{4}$ & $6.1 \times 10^{3}$ & $4.8 \times 10^{3}$ & $7.4 \times 10^{3}$ & 433 \\
$30 \mathrm{~S}$ & $3.9 \times 10^{5}$ & $5.0 \times 10^{5}$ & $2.3 \times 10^{4}$ & $4.4 \times 10^{4}$ & $3.3 \times 10^{4}$ & 83 \\
\hline
\end{tabular}

\section{SUPERRADIANT POPULATION TRANSFER}

We have argued above that the transfer mechanism responsible for population transfer out of the excitation state in a few microseconds cannot be due to nearresonant energy transfer collisions between excited Rydberg atoms. L-changing collisions between Rydberg atoms and free electrons are another possible mechanism. Such collisions are unlikely to be energetic enough for the recoil to eject atoms from the trap and so would agree with much of the observed behavior. However, at our low excitation rates it is unlikely that electrons are present in sufficient quantities to cause the observed state transfer. With black-body ionization rates of 400/s (see Section VI) acting on a population of $10^{4}$ Rydberg atoms, free electrons are produced at a rate of $4 \times 10^{6} / \mathrm{s}$. Free electrons are typically fast-moving and dissipate at a rate of $\sim 10^{4} / \mathrm{s}$ [33], giving an average free electron population of $\sim 400$, which would not have a large impact on the $10^{4}$ atoms in the excitation state. We estimate that there is insufficient ionization to cause an electron trap [33. In addition, the rate of l-changing collisions should increase with principle quantum number, in contrast with our observations.

Having argued against collisional phenomena being responsible for Rydberg energy transfer, we need a radiative mechanism to explain our results. Recently, Wang et al. [28] observed superradiance in the measured lifetimes of Rydberg states. That superradiance could play an important role in Rydberg population dynamics can be understood by the following arguments. For principal quantum numbers $>20$, the size of the MOT is less than the wavelength for radiative emission from the excitation state to nearby dipole-allowed states. If there are $N$ 
atoms initially in a particular Rydberg state, the collective dipole moment is enhanced by a factor of $N$ (assuming the atom cloud is much smaller than the wavelength of the emitted light). The emission rate is enhanced by a factor of $N^{2}$, or by a factor of $N$ on a per-atom basis. Since $N$ is on the order of $10^{4}$ for our experiment, the superradiant decay rate will be on the order of

$$
\frac{2}{3} \frac{\omega^{3} N d^{2}}{\hbar c^{3}} \sim \frac{4 N}{3 n^{5}} \alpha^{3} \frac{R y}{\hbar}
$$

which is $3 \times 10^{5} / \mathrm{s}$ for $10^{4} n=50$ Rydberg atoms. The low emission frequency is compensated for by the large number of cooperatively radiating atoms. Since the spontaneous decay rate is proportional to $n^{-3}$, the relative importance of superradiance and spontaneous decay goes only as $n^{-2}$, a factor of only 4 for this experiment.

Wang et al. 28] developed a sophisticated theory of superradiance with application to cold Rydberg gases. Here we present a simplified model of superradiance to use as a interpretive guide. We base this model on Dicke's original work 34 as elucidated by Gross and Haroche 35] and Rehler and Eberly [36].

In considering the superradiant decay of an initial state $|e\rangle$ to a lower energy state $|l\rangle$, the Dicke approach introduces an effective collective spin state of the $2 \mathrm{~N}$-level system as $|J M\rangle$, with $N_{e}=J+M$ atoms in state $|e\rangle$ and $N_{l}=J-M$ atoms in state $|l\rangle$. The radiation rate is found to be $\Gamma_{e l}(J(J+1)-M(M-1))=\Gamma_{e l} N_{e}\left(N_{l}+1\right)$. To extend the two-level case to our multi-level case, we assume that we can model superradiance with a set of rate equations

$$
\frac{d N_{e}}{d t}=-\sum_{l<e} \Gamma_{e l} N_{e}\left(N_{l}+1\right)+\sum_{l^{\prime}>e} \Gamma_{l^{\prime} e} N_{l^{\prime}}\left(N_{e}+1\right)
$$

This model reproduces the key features of superradiance: a large initial inversion radiates at $\Gamma_{e l}$ (per atom) at first, then as the inversion is reduced the rate accelerates to a maximum of $N \Gamma_{e l}^{\prime} / 4$, occurring over a time $(\ln N) /\left(\Gamma_{e l} N\right) \ll 1 / \Gamma_{e l}$ 36]. As an additional check on the model, we have simulated the experiment of Ref. 37. (which was performed at high temperatures and much smaller $n$ ) and our model reproduces the dominant features of the data shown there.

The emission rates $\Gamma_{e l}$ are the rates for spontaneous emission multiplied by a cooperativity parameter $C_{e l}$ :

$$
\Gamma_{e l}=C_{e l} \frac{2 e^{2} \omega_{e l}^{3}}{m c^{3}} \frac{\left(2 J_{l}+1\right)}{\left(2 J_{e}+1\right)} f_{e l}
$$

where the $f_{e l}$ are the calculated absorption oscillator strengths. For a system of atoms whose spatial extent is on the order of the wavelength of the transition, superradiance will occur at a reduced rate. The wavelength of the transition to the nearest lower lying Rydberg state varies from $0.17 \mathrm{~cm}$ for $30 \mathrm{~S}-29 \mathrm{P}$ to $2.8 \mathrm{~cm}$ for the $58 \mathrm{D}$ $59 \mathrm{P}$ transition. For low $n$, this is quite close to the $1 \mathrm{~mm}$ spatial extent of the MOT, and lower levels will have even shorter transition wavelengths. Following Ref. [36], the cooperativity parameter for a uniform density system of $\mathrm{N}$ atoms in a volume $\mathrm{V}$ radiating in direction $\hat{k}$ is (in the $N \gg 1$ limit)

$$
\begin{aligned}
C_{e l} & =\frac{1}{V^{2}} \int d^{3} x \int d^{3} x^{\prime} e^{i\left(\vec{k}-\vec{k}_{1}\right) \cdot\left(\vec{x}-\vec{x}^{\prime}\right)} \\
& =\frac{9\left(\sin \left(k_{e l} R\right)-k_{e l} R \cos \left(k_{e l} R\right)\right)^{2}}{\left(k_{e l} R\right)^{6}},
\end{aligned}
$$

for a spherical uniform density cloud, where $k R$ is the product of the wavenumber and the radius of the atomic sample. The cooperativity parameter is 1 for $R \ll \lambda$ and decreases to 0 for $R \gg \lambda$. In practice, the cooperativity parameter becomes small around $n=20$ for a $1 \mathrm{~mm}$ MOT.

To account for superradiance, we replace the level $|s\rangle$ of the model of Section IV with a set of levels near in energy to $|r\rangle$ that are coupled to each other and to $|r\rangle$ by black-body radiation and by superradiance from Eq. 11. We find steady-state solutions of the resulting non-linear equations and from them deduce the effective RydbergRydberg transfer rate from Eq. 11] with $e=r$.

Table II compares the observed Rydberg transfer rates and those predicted by our simulation. These rates do not drop off as quickly as would be expected from Equation 10. This is a result of the spatial factor approaching unity for the higher n-levels, balancing out the decrease in natural emission rate. Additionally as a result of this increasing spatial factor, our simulation indicates that the atoms are transferred predominantly to f-states for the 43d and 58d levels. This transfer to f-states could explain the higher total loss rate from the trap for these levels. We emphasize that there are no adjustable parameters in our simplified superradiance model, and yet it naturally predicts the order of magnitude of the Rydberg-Rydberg transfer rates. A more sophisticated model would be expected to explain the variation seen.

TABLE II: A comparison of Rydberg-Rydberg transfer rates deduced by comparing measurements deduced from the model of Section IV with those predicted by the superradiance model. All units are $\mathrm{s}^{-1}$.

\begin{tabular}{ccc}
\hline \hline State & $\gamma$ (calculated $)$ & $\gamma($ expt $)$ \\
\hline 28D & $1.7 \times 10^{5}$ & $1.3 \times 10^{5}$ \\
43D & $2.4 \times 10^{5}$ & $7.4 \times 10^{4}$ \\
58D & $1.2 \times 10^{5}$ & $2.0 \times 10^{4}$ \\
30S & $2.2 \times 10^{5}$ & $5.0 \times 10^{5}$ \\
\hline
\end{tabular}

\section{BLACK-BODY IONIZATION}

So far we have not discussed the actual mechanism for trap loss. The deduced trap loss rates are quite modest, typically $400 / \mathrm{s}$ on a per Rydberg atom basis. This is 
close to what would be expected from black-body ionization. Black-body ionization rates were recently calculated in Ref. [38] for the various Rydberg levels. Assuming Rydberg $n$-level distributions from our superradiance model, we find expected photoionization rates from black-body radiation to be as shown in Table III. For the $\mathrm{D}$ states, there is reasonable agreement between our deduced experimental rates and the predicted values, suggesting that black-body ionization probably composes a large portion of the total loss from the trap. For the sstate, the predicted trap loss is greater than observed by about a factor of 3 , for which we have no explanation.

TABLE III: Comparison of inferred Rydberg trap loss rates with black-body ionization rates. $\Gamma_{B B I}$ is the rate of blackbody ionization for each of these states calculated from Ref. [38]. All rates are in units of $\mathrm{s}^{-1}$.

\begin{tabular}{ccc}
\hline \hline State & $\Gamma_{\text {s calc }}$ & $\Gamma_{B B I}$ \\
\hline $28 \mathrm{D}$ & 212 & 322 \\
$43 \mathrm{D}$ & 470 & 720 \\
$58 \mathrm{D}$ & 329 & 457 \\
$30 \mathrm{~S}$ & 77 & 265 \\
\hline
\end{tabular}

\section{DISCUSSION}

In this paper, we have presented results on the dynamics of low-density ultracold Rydberg clouds excited using two-photon absorption from a MOT. By looking at trap loss, radiative cascade, and state-selective stimulated emission we find that the probability of the Rydberg atoms undergoing collisional loss before decaying back to the ground state is small. Additionally, the Rydberg state produced by the two-photon excitation is depleted by some mechanism on a time scale significantly shorter than can be explained by black-body transfer or single-atom radiative decay. This time scale increases with increasing principal quantum number, in contradic- tion to expectations if inelastic Rydberg-Rydberg collisions were responsible for the rapid state transfer. On the other hand, estimates of collective superradiant light emission and a simplified model thereof suggest that this mechanism can explain the rapid population transfer observed in this experiment. The overall trap loss rates are consistent with expectations from black-body ionization.

Before the experiment of Wang et al. [28] the effects of superradiance were not appreciated for the population dynamics of high density ultracold Rydberg atoms. Superradiance can happen on very rapid time scales, especially under strong Rydberg excitation as achieved in a number of previous experiments. In fact, superradiance can easily occur on sub-microsecond time scales. A key consequence of superradiance is that it populates Rydberg states lying energetically below the state being excited by the laser. Indeed, in the experiments of Ref. [13] and Ref. [39], population clearly moves to lower lying Rydberg levels on a fast time scale, consistent with the hypothesis of superradiant transfer.

An additional consequence of fast superradiant population transfer is that it provides a mechanism for rapid population of states of neighboring orbital angular momentum $l$ from the excitation state. Pairs of atoms with $\delta l= \pm 1$ interact at long range via the $R^{-3}$ resonant dipole-dipole interaction, not the usual $R^{-6}$ van der Waals interaction. The much stronger collision interactions between these atoms may explain the very rapid time scales for plasma formation in a number of experiments where resonant dipole-dipole interactions were not purposely produced using external fields.

\section{Acknowledgments}

J. Strabley contributed to the early stages of this work. We appreciate helpful discussions with M. Saffman and D. Yavuz. This work was supported by the National Science Foundation and NASA.
[1] I. Mourachko, D. Comparat, F. de Tomasi, A. Fioretti, P. Nosbaum, V. M. Akulin, and P. Pillet, Phys. Rev. Lett. 80, 253 (1998).

[2] W. R. Anderson, J. R. Veale, and T. F. Gallagher, Phys. Rev. Lett. 80, 249 (1998).

[3] S. Farooqi, D. Tong, S. Krishnan, J. Stanojevic, Y. Zhang, J. Ensher, A. Estrin, C. Boisseau, R. Cote, E. Eyler, et al., Phys. Rev. Lett. 91, 183002 (2003).

[4] K. Afrousheh, P. Bohlouli-Zanjani, D. Vagale, A. Mugford, M. Fedorov, and J. D. D. Martin, Phys. Rev. Lett. 93, 233001 (2004).

[5] K. Afrousheh, P. Bohlouli-Zanjani, J. D. Carter, A. Mugford, and J. D. D. Martin, Phys. Rev. A 73, 063403 (2006).

[6] T. Vogt, M. Viteau, J. Zhao, A. Chotia, D. Comparat, and P. Pillet, Phys. Rev. Lett. 97, 083003 (2006).

[7] P. Bohlouli-Zanjani, J. A. Petrus, and J. D. D. Martin, Phys. Rev. Lett. 98, 203005 (2007).

[8] W. Li, P. J. Tanner, and T. F. Gallagher, Phys. Rev. Lett. 94, 173001 (2005).

[9] K. Singer, M. Reetz-Lamour, T. Amthor, S. Fölling, M. Tscherneck, and M. Weidemüller, J. Phys. B 38, S321 (2005).

[10] T. Amthor, M. Reetz-Lamour, S. Westermann, J. Denskat, and M. Weidemüller, Phys. Rev. Lett. 98, 023004 (2007).

[11] T. J. Carroll, S. Sunder, and M. W. Noel, Phys. Rev. A 73, 032725 (2006).

[12] M. P. Robinson, B. L. Tolra, M. W. Noel, T. F. Gallagher, and P. Pillet, Phys. Rev. Lett. 85, 4466 (2000). 
[13] A. Walz-Flannigan, J. R. Guest, J. H. Choi, and G. Raithel, Phys. Rev. A 69, 063405 (2004).

[14] D. Jaksch, J. I. Cirac, P. Zoller, S. L. Rolston, R. Côté, and M. D. Lukin, Phys. Rev. Lett. 85, 2208 (2000).

[15] M. D. Lukin, M. Fleischhauer, R. Cote, L. M. Duan, D. Jaksch, J. I. Cirac, and P. Zoller, Phys. Rev. Lett. 87, 037901 (2001).

[16] J. Sebby-Strabley, R. T. R. Newell, J. O. Day, E. Brekke, and T. G. Walker, Phys. Rev. A 71, 021401(R) (2005).

[17] D. D. Yavuz, P. B. Kulatunga, E. Urban, T. A. Johnson, N. Proite, T. Henage, T. G. Walker, and M. Saffman, Phys. Rev. Lett. 96, 063001 (2006).

[18] E. Brion, K. Molmer, and M. Saffman, Phys. Rev. Lett. 99, 260501 (pages 4) (2007).

[19] M. Saffman and T. G. Walker, Phys. Rev. A 66, 065403 (2002).

[20] M. Saffman and T. G. Walker, Phys. Rev. A 72, 042302 (2005).

[21] T. G. Walker and M. Saffman, arXiv:0712.3438v1 [quantph] (2008).

[22] R. Heidemann, U. Raitzsch, V. Bendkowsky, B. Butscher, R. Löw, L. Santos, and T. Pfau, Phys. Rev. Lett. 99, 163601 (2007).

[23] K. Singer, M. Reetz-Lamour, T. Amthor, L. G. Marcassa, and M. Weidemüller, Phys. Rev. Lett. 93, 163001 (2004).

[24] D. Tong, S. M. Farooqi, J. Stanojevic, S. Krishnan, Y. P. Zhang, R. Cote, E. E. Eyler, and P. L. Gould, Phys. Rev. Lett. 93, 063001 (2004).

[25] T. C. Liebisch, A. Reinhard, P. R. Berman, and G. Raithel, Phys. Rev. Lett. 95, 253002 (2005), Phys. Rev. Lett. 98, 109903(E) (2007).

[26] T. A. Johnson, E. Urban, T. Henage, L. Isenhower, D. D. Yavuz, T. G. Walker, and M. Saffman, arXiv:0711.0401
(2008)

[27] R. Heidemann, U. Raitzsch, V. Bendkowsky, B. Butscher, R. Low, and T. Pfau, Phys. Rev. Lett. 100, 033601 (2008).

[28] T. Wang, S. F. Yelin, R. Côté, E. E. Eyler, S. M. Farooqi, P. L. Gould, M. Koštrun, D. Tong, and D. Vrinceanu, Phys. Rev. A 75, 033802 (pages 4) (2007).

[29] S. K. Dutta, D. Feldbaum, A. Walz-Flannigan, J. R. Guest, and G. Raithel, Phys. Rev. Lett. 86, 3993 (2001).

[30] A. Reinhard, T. C. Liebisch, B. Knuffman, and G. Raithel, Phys. Rev. A 75, 032712 (2007).

[31] B. K. Teo, D. Feldbaum, T. Cubel, J. R. Guest, P. R. Berman, and G. Raithel, Phys. Rev. A 68, 053407 (2003).

[32] T. F. Gallagher, Rydberg Atoms (Cambridge University Press, 1994).

[33] T. C. Killian, S. Kulin, S. D. Bergeson, L. A. Orozco, C. Orzel, and S. L. Rolston, Phys. Rev. Lett. 83, 4776 (1999).

[34] R. H. Dicke, Phys. Rev. 93, 99 (1954).

[35] M. Gross and S. Haroche, Phys. Rep. 93 (1982).

[36] N. E. Rehler and J. H. Eberly, Phys. Rev. A 3, 1735 (1971).

[37] P. R. F. F Gounand, M Hugon and J. Berlande, Journal of Physics B: Atomic and Molecular Physics 12, 547 (1979), URL http://stacks .iop.org/0022-3700/12/547.

[38] I. I. Beterov, D. B. Tretyakov, I. I. Ryabtsev, A. Ekers, and N. N. Bezuglov, arXiv.org:physics/0702192 (2007).

[39] W. Li, M. W. Noel, M. P. Robinson, P. J. Tanner, T. F. Gallagher, D. Comparat, B. Laburthe Tolra, N. Vanhaecke, T. Vogt, N. Zahzam, et al., Phys. Rev. A 70, 042713 (2004). 